\title{
Analysis of the prevalence and influencing factors of depression and anxiety among maintenance dialysis patients during the COVID-19 pandemic
}

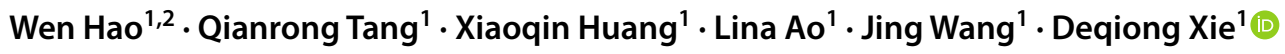

Received: 20 August 2020 / Accepted: 30 January 2021 / Published online: 6 March 2021

(c) The Author(s), under exclusive licence to Springer Nature B.V. part of Springer Nature 2021

\begin{abstract}
Objective Currently, the COVID-19 outbreak and its spread around the globe is significantly affecting mental health and health in general, worldwide. During the COVID-19 pandemic, the general medical complications have received the most attention, whereas only a few studies address the potential direct impact of SARS-CoV-2 on mental health.

Methods A total of 321 maintenance hemodialysis (MHD) patients were selected using random sampling from the hemodialysis center of the second people's Hospital of Yibin. They completed Zung's self-rating anxiety scale (SAS) for anxiety, Zung's self-rating depression scale (SDS) for depression, and the activity of daily living scale (ADL) for the ability of living. Demographic data and laboratory tests were used to analyze the risk factors.

Results The proportions of the prevalence of anxiety and depression symptoms were between $34.89 \%$ and $30.02 \%$, respectively, among the MHD patients. The SAS and SDS scores of the 321 patients were $45.42( \pm 10.99)$ and $45.23( \pm 11.59)$, respectively. The results show that monthly income, medical insurance, vascular access, the duration of dialysis, complication, hemoglobin (HGB), immunoreactive parathyroid hormone (iPTH) and blood phosphorus (P) are factors that influence anxiety among patients ( $p<0.05$ for all). Vascular access, monthly income, medical insurance, complication, CRP, Alb, are factors that influence depression among patients $(p<0.05$ for all).

Conclusion Our results suggest that during the pandemic period, the prevalence of anxiety and depression symptoms among MHD patients increased. Some demographic and clinical variables were associated with it. We should, therefore, pay more attention to the patients' psychology, start targeted intervention to alleviate the patients' anxiety and further improve their quality of life.
\end{abstract}

Keywords COVID-19 $\cdot$ Anxiety $\cdot$ Depression $\cdot$ Maintenance hemodialysis $\cdot$ Logistic models

\section{Introduction}

The recent worldwide COVID-19 outbreak is a grave infectious disease, lacks specific and effective treatment methods and is difficult to prevent and control [1,2]. This large scale, infectious and public health event has caused other health problems such as stress, anxiety, depression, insomnia, denial, anger, and fear worldwide. Recent studies show that during the COVID-19 outbreak, the depression rate in the

Deqiong Xie

1285396756@qq.com

1 Department of Nephrology, The Second People's Hospital of Yibin, Yibin 644000, Sichuan, China

2 North Sichuan Medical College, Nanchong, Sichuan, China general global population ranged from $3.7 \%$ to $48.3 \%$. The proportion of the prevalence of depression symptoms was $6.21 \%$ and $6.46-50.7 \%$ among quarantined individuals and medical workers in China, respectively. However, to date, no detailed study on the mental health status of maintenance hemodialysis (MHD) patients facing the epidemic has been conducted.

During the epidemic, MHD patients were considered a special group following their regular dialysis and frequent visits to the hospital blood purification center. Most of the patients are middle-aged and elderly, and often have hypertension, diabetes and other diseases; due to their generally lower immune function, they are the most susceptible to COVID-19 [3]. Further, they are treated in a relatively crowded space which increases their psychological burden. Although the National Health Service Committee has issued the Novel Coronavirus Infection 
Emergency Guidelines for Psychological Crisis Intervention as a guide to psychological intervention and psychological counseling, psychological problems of dialysis patients are still given significantly insufficient attention. Although several studies have investigated anxiety and depression among diabetes and cancer patients and the general population of China, none of these studies focused on MHD patients. This study aims to ascertain the risk factors of anxiety among dialysis patients and further provide a theoretical basis for the implementation of effective measures and psychological intervention.

\section{Objective and methods}

\section{Study population and sample}

This study is a cross-sectional survey. The target population includes long-term dialysis patients from February 2013 to March 2020 in the Second People's Hospital of Yibin City. We used structured questionnaires to assess the mental health of these patients during the COVID-19 outbreak. The survey ran from March 20 to March 29, 2020. During the investigation and research period, China established and implemented strict prevention and control measures nationwide, such as wearing masks, isolating at home, and limiting hospital companions. The local government has also imposed restrictions on hospitals with dialysis patients, including a series of measures for blood purification centers, such as setting up a prevention and treatment team, formulating new rules and regulations for coronary heart disease infection, recommending the abolition of escorts, dividing the function of hemodialysis rooms. Inclusion criteria: (a) maintenance hemodialysis/peritoneal dialysis patients; (b) agree to participate in the survey; (c) able to read or understand the contents of the questionnaire and communicate orally or through writing. Exclusion criteria: (a) patients with a history of mental and neurological diseases, long-term alcohol or drug abuse, and long-term use of opioid or valium drugs; (b) patients with unclear awareness, cognitive impairment or serious audio-visual impairment; (c) patients with complications that may affect the accuracy of the scale evaluation: patients with serious heart, liver, brain and other diseases; (d) unable to adhere to the rules of the survey or answer the questionnaire. This study was approved by the ethics committee of the Second People's Hospital of Yibin, where all the participants signed informed consent.

\section{Methods}

\section{Rating instruments}

Self-made general demographic data questionnaire: we referred to relevant literature to design a general demographic data questionnaire which includes questions on gender, age, monthly income, medical insurance, duration of dialysis, vascular access, complication, worry about the prognosis of the COVID-19 infection, among others.

Self-rating anxiety scale: Zung's self-rating anxiety scale (SAS) [4] was used to assess anxiety symptoms. The SAS was compiled by W. K. Zung in 1971; it is reliable, valid, and has a wide range of applications. The Cronbach $\alpha$ coefficient is 0.823 . This scale contains 20 items that reflect on anxiety subjective feelings. Each item is divided into four grades: 15 are positive scores and 5 are reverse scores (5t, 9, 13, 17, 19 questions). The respondents scored each item ranging from 1 (none or small) to 4 (most or all times) based on how they felt over the past week. According to the results of the Chinese norm, the standard, mild, moderate, and severe anxiety score points are 50,50-59, 60-69, and above 69 , respectively.

Self-rating depression scale: Zung's self-rating depression scale (SDS) was used to evaluate the symptoms of depression. This scale consists of 20 items. Each set of 10 items indicate negative and positive experiences with a 4-point Likert scale ranging from 1 (none or few times) to 4 (most or all times). In this study, the cut-off point for the symptoms of depression was based on an index score of 50 .

\section{Investigation methods}

The researchers delivered the mental health assessment questionnaire to the participants face-to-face. Each participant filled out a detailed questionnaire on WeChat (a widely used social media application in China), including SAS, SDS, and the general demographic data questionnaire, to reduce the possibility of contact with those participants who may have been infected by COVID-19. The participants further provided general information and social characteristics, including age, gender, medical insurance, marriage, dialysis time, complications, etc. Further, we individually explained the process and guided the elderly who were unable to fill in the questionnaire online, and those patients with low education and ability to understand the questionnaires' contents. The questionnaires were kept anonymous to ensure the confidentiality and reliability of the data. In conclusion, a total of 326 patients participated in the study. Five of the patients were excluded as per the inclusion/exclusion criteria. Further, 321 respondents who completed the questionnaire were included in the final analysis ( $98.47 \%$ response rate).

\section{Data analysis}

The Statistical Package for Social Sciences (SPSS) version 22.0 was used to analyze all the data. Continuous data were expressed as mean $\pm \mathrm{SD}$. The single factor analysis of variance was used to compare the between-group differences. 
Statistically significant variables were screened and included in multivariate logistic regression analyses. The estimates of the strengths of associations were demonstrated by the odds ratio (OR) with a 95\% confidence interval (CI). Spearman's correlation coefficient $(r)$ was used to evaluate the association between laboratory inspection data and anxiety and depression levels. $p$-values of less than 0.05 were considered statistically significant (2-sided tests).

\section{Results}

\section{Demographic and clinical parameters}

Three hundred and twenty-one participants were included in this study, including 188 men (58.57\%), 133 women (41.43\%), 188 patients between 18 and 65 years $(58.57 \%)$ and 184 patients whose monthly income was less than 5,000 yuan $(57.32 \%), 215$ patients (66.98\%) that underwent dialysis for more than 1 year, 240 patients $(74.77 \%)$ of vascular access by arteriovenous fistula, of which there were 55 cases $(17.13 \%)$ with cardiovascular and cerebrovascular complications. The average hemoglobin (HGB) was 102.59 $( \pm 18.28) \mathrm{g} / \mathrm{L}$, albumin (Alb) $37.43( \pm 4.45) \mathrm{g} / \mathrm{L}$, and neutrophils/lymphocytes (NLR) $4.70( \pm 2.79)$, intact parathyroid hormone (iPTH) $612.80( \pm 414.04) \mathrm{pg} / \mathrm{mL}$, blood phosphorus $(\mathrm{P}) 1.78( \pm 0.66) \mathrm{mmol} / \mathrm{L}, \beta 2$ microglobulin 31.34 $( \pm 7.63) \mathrm{mg} / \mathrm{L}$, C-reactive protein $(\mathrm{CRP}) 5.97( \pm 7.05) \mathrm{mg} / \mathrm{L}$, RBC width standard $49.40( \pm 5.30) \mathrm{fL}$. The results showed that dialysis patients were generally anemic, with low Alb, high P, iPTH, and CRP, and further showed a micro-inflammatory state.

\section{Analysis of anxiety and depression symptoms of MHD patients}

Among the 321 MHD patients, the SAS and SDS scores of MHD patients were $(45.42 \pm 10.99)$ and $(45.23 \pm 11.59)$, respectively. There were 112 cases $(34.89 \%)$ of MHD patients with anxiety, including 79 (70.53\%), 30 (26.97\%), $3(2.78 \%)$, and 103 (32.09\%) cases of patients with mild, moderate, and severe anxiety and depression, respectively. Further, 69 (66.99\%), 32 (31.07\%) and 2 (1.94\%) of these cases were of patients with mild, moderate, and severe depression, respectively.

\section{Single factor analysis of anxiety and depression among MHD patients}

The general information and laboratory tests of the MHD patients were divided into groups according to whether they were anxious or depressed. The monthly incomes, medical insurances, vascular access, duration of dialysis, open psychological special line, worry about the prognosis of COVID-19 infection, complication, and HGB, Alb, NLR, iPTH, P, and CRP of the anxiety and non-anxiety groups (all $p<0.05$ ) differed significantly. Further, Figs. 1, 2 show that the monthly income, medical insurance, vascular access, duration of dialysis, worry about prognosis, complication, and HGB, Alb, NLR, iPTH, P, and CRP of the depression and non-depression groups (all $p<0.05$ ) differed significantly.

\section{Correlation between SAS/SDS scores and laboratory tests among MHD patients}

The Pearson correlation analysis results showed that the SAS and SDS scores of MHD patients were positively correlated with HGB, Alb, iPTH, P, NLR, CRP, ADL, and SDS (all $p<0.05$ ), as shown in Table 1.

\section{Analysis of multiple factors influencing the SAS and SDS scores of MHD patients}

The above statistically significant factors were taken as independent variables, and whether anxiety symptoms were set as dependent variables for stepwise regression analysis. The results showed that dialysis duration, vascular access, medical insurance, monthly income, complications, iPTH, $\mathrm{P}$ and HGB were factors that influence anxiety $(p<0.05)$. The risk of anxiety among dialysis patients with a monthly income of less than 5,000 RMB was 4.223 times higher than that in patients with a monthly income of more than 5,000 $\mathrm{RMB}(\mathrm{OR}=4.223,95 \% \mathrm{CI}=2.045-8.720)$. The risk of anxiety (Fig. 3) among patients who underwent dialysis for less than one year is 2.018 times that of patients who underwent dialysis for more than one year $(\mathrm{OR}=2.018$, 95\% CI $=1.106-3.681$ ). Furthermore, the risk of anxiety among patients with a residential medical insurance is 0.031 times that of patients who self-payed $(\mathrm{OR}=0.031$, $95 \% \mathrm{CI}=0.009-0.105$ ), while the risk of anxiety for employees with a medical insurance is 0.048 times that of those opting for self-pay (OR $=0.048,95 \% \mathrm{CI}=0.015-0.149)$. The risk of anxiety among patients without health complications (Fig. 4) was 0.384 times that of patients with health complications $(\mathrm{OR}=0.384,95 \% \mathrm{CI}=0.184-0.802)$. Further, the risk of anxiety increased by $0.1 \%(\mathrm{OR}=1.001$, $95 \% \mathrm{CI}=1.001-1.002$ ) for every unit increase in the $\mathrm{iPTH}$ value of MHD patients. The risk of anxiety increased by $70 \%$ ( $\mathrm{OR}=1.700,95 \% \mathrm{CI}=1.135-2.546)$ for every unit increase in $\mathrm{P}$. However, the risk of anxiety decreased by $2.3 \%(\mathrm{OR}=0.967,95 \% \mathrm{CI}=0.952-0.982)$ for each unit increase in the HGB, as shown in Fig. 5.

The factors with statistical significance in univariate analysis were taken as independent variables, and whether depression symptom was set as dependent variables for 

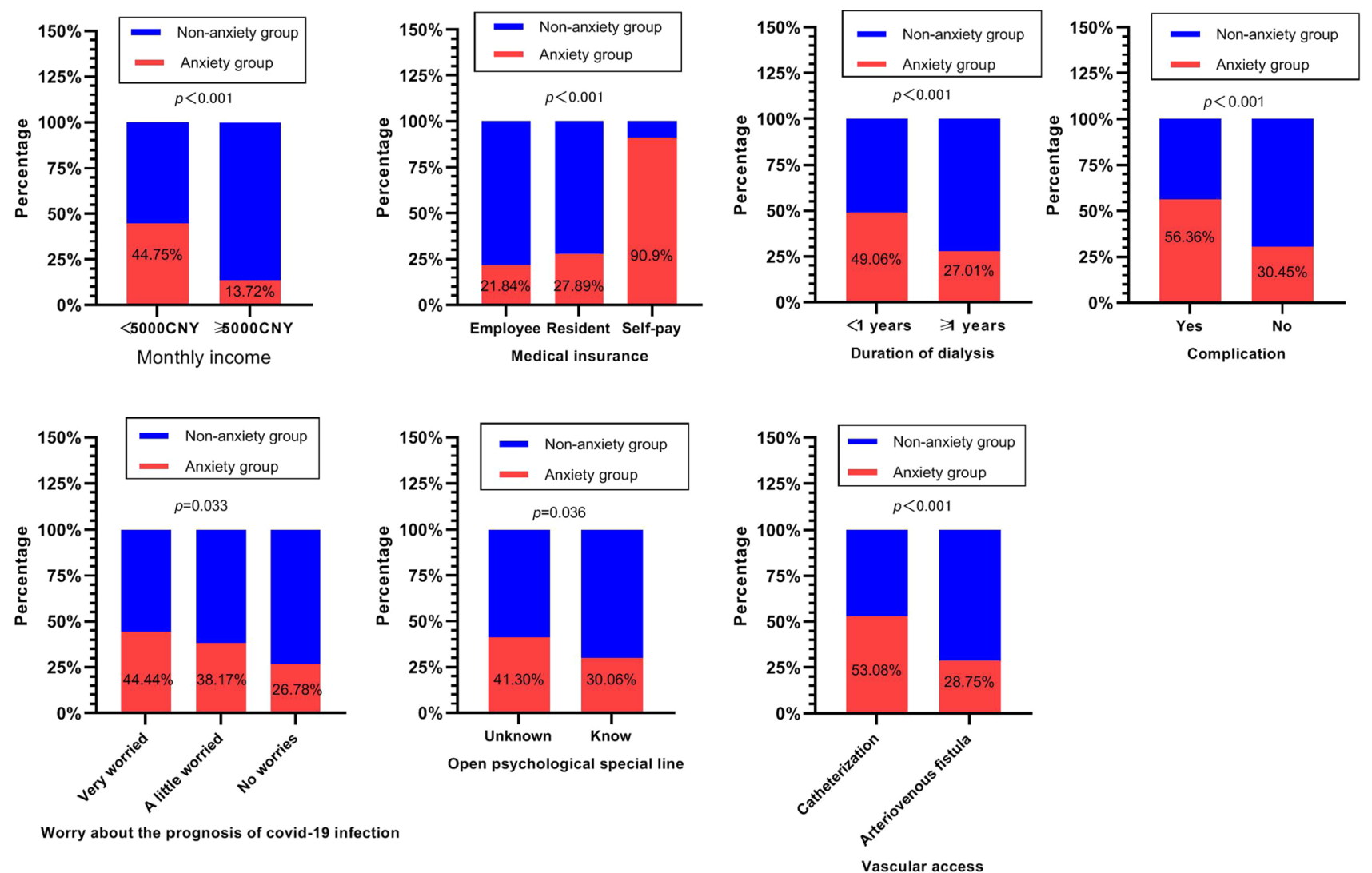

Fig. 1 Single-factor analysis of anxiety among MHD patients with different demographic characteristics $(N=321)$
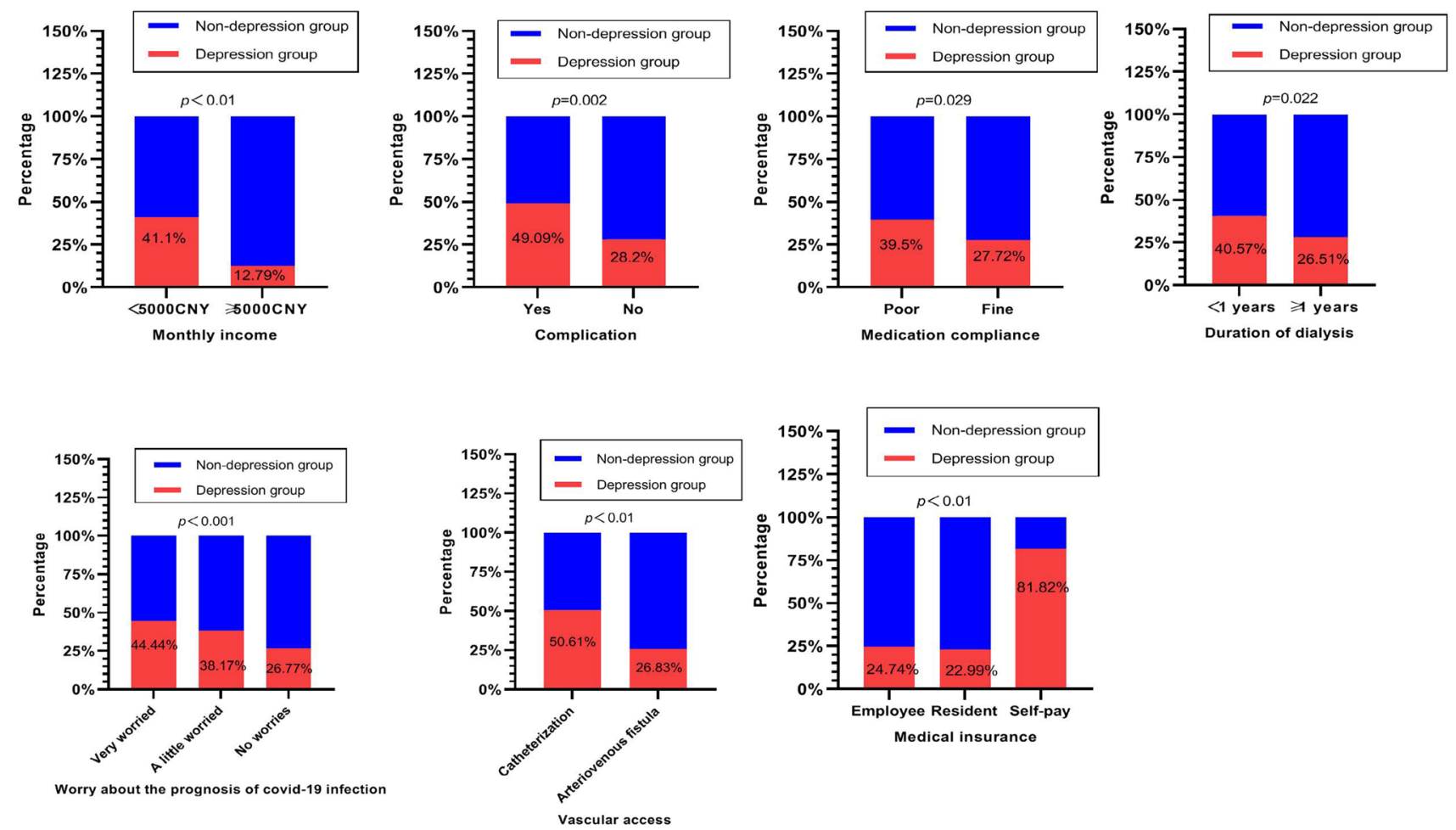

Fig. 2 Single-factor analysis of depression among MHD patients with different demographic characteristics $(N=321)$ 
Table 1 Pearson correlation analysis between SAS/ SDS score and laboratory examination of MHD patients $(N=321)$

\begin{tabular}{lllllllll}
\hline Variables & HGB & Alb & iPTH & P & NLR & CRP & ADL & SAS/SDS \\
\hline $\begin{array}{l}\text { Mean value } \\
\text { SAS }\end{array}$ & $103 \pm 17$ & $37.5 \pm 3.9$ & $638 \pm 394$ & $1.8 \pm 0.6$ & $4.7 \pm 2.8$ & $6.2 \pm 6.7$ & $19.0 \pm 6.0$ & \\
$r$ & & & & & & & & \\
$p$ & -0.176 & -0.142 & 0.167 & 0.324 & 0.188 & 0.156 & 0.332 & 0.927 \\
SDS & 0.002 & 0.011 & 0.003 & 0.000 & 0.001 & 0.005 & 0.000 & 0.000 \\
$r$ & & & & & & & & \\
$p$ & -0.166 & -0.138 & 0.135 & 0.299 & 0.194 & 0.139 & 0.303 & 0.927 \\
\hline
\end{tabular}
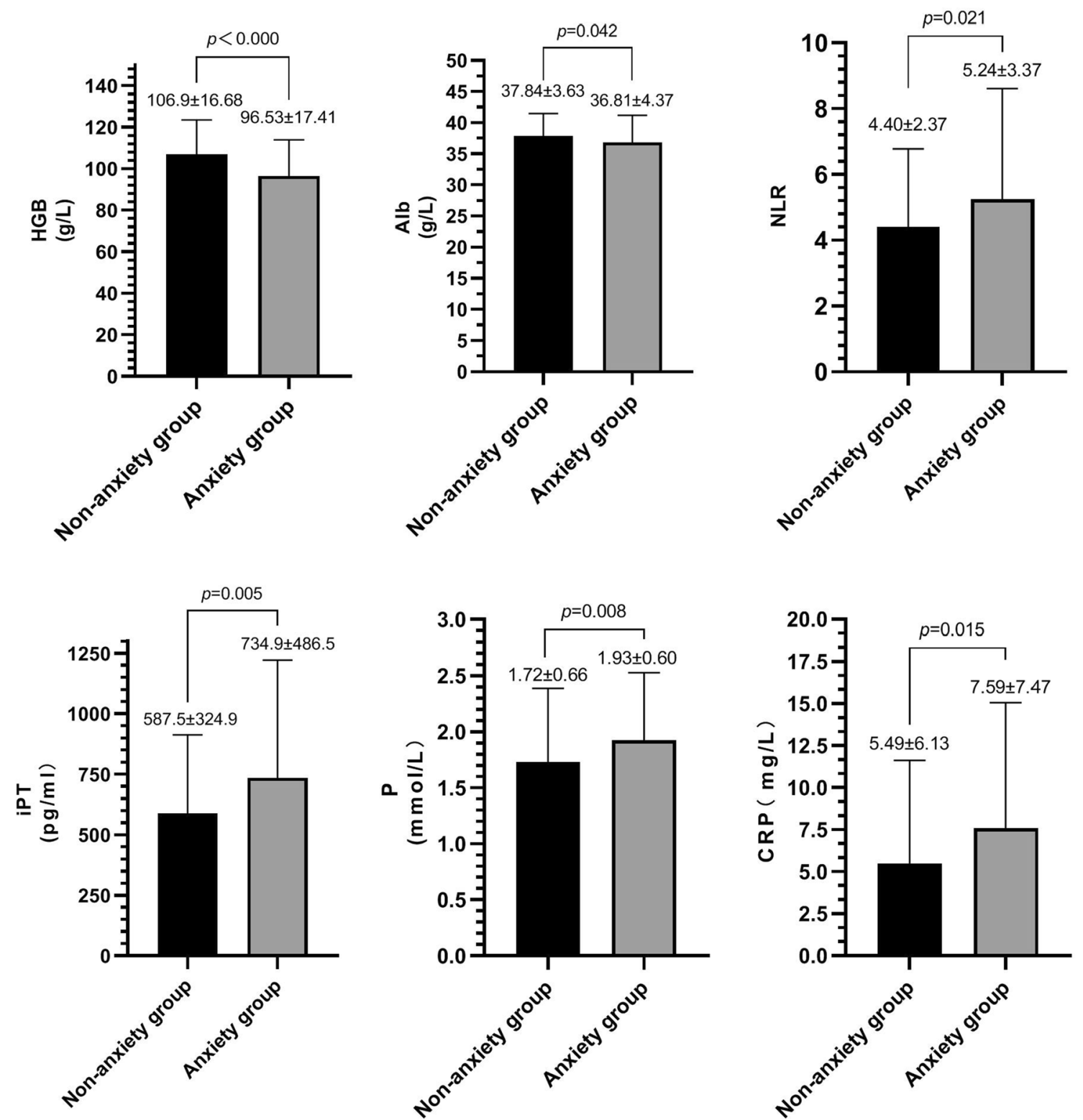

Fig. 3 Single-factor analysis of anxiety among MHD patients with laboratory tests $(N=321)$ 

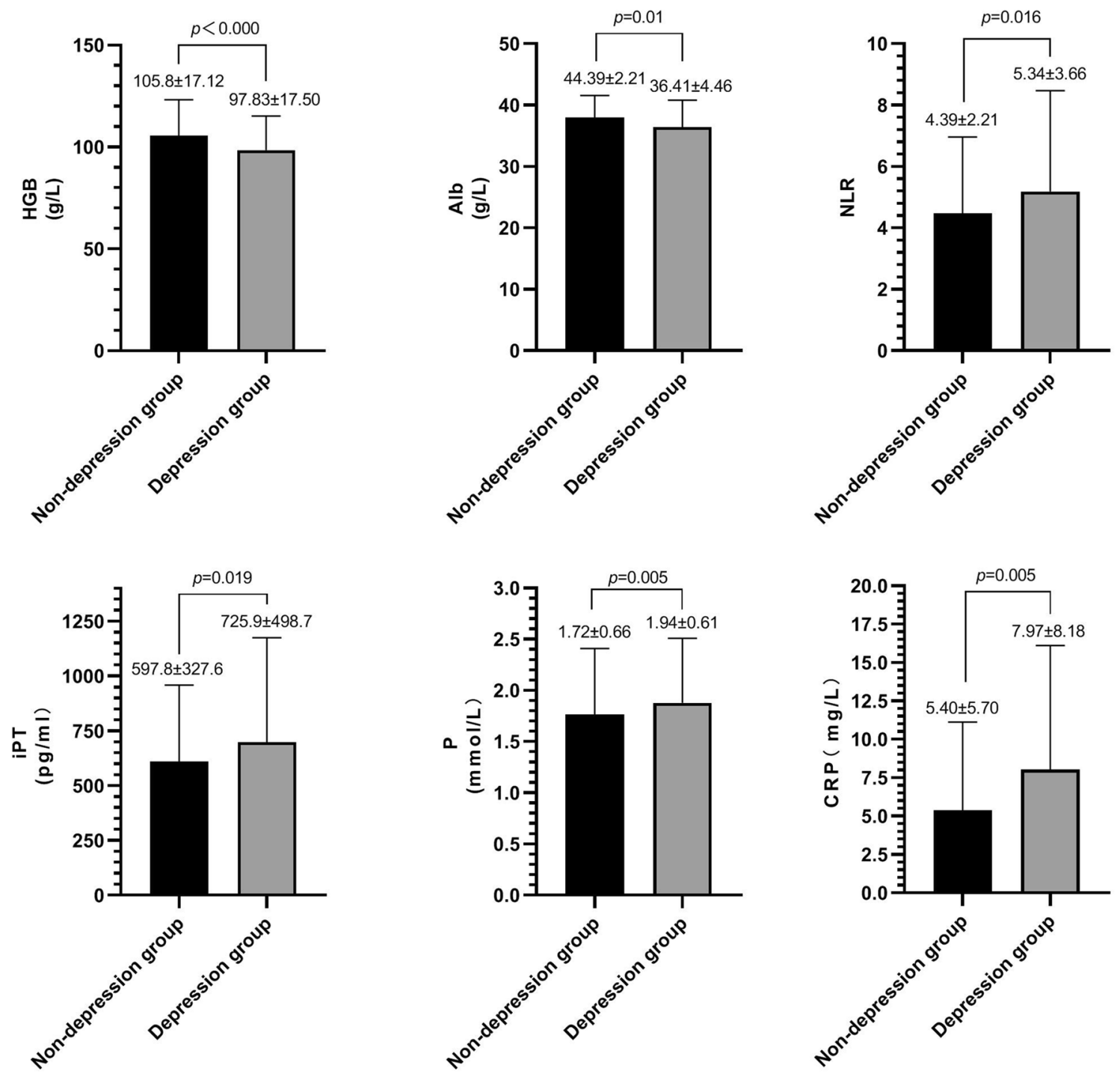

Fig. 4 Single-factor analysis of depression among MHD patients with laboratory tests $(N=321)$. $S D$ standard deviation, $S D S$ Zung's selfrating depression scale, SAS Zung's self-rating anxiety scale. Normal value range of each index: HGB (men 131-172, women 113-151), iPTH 12-88, P 0.8-1.45, Alb 40-55, CRP 0.00-10 mg/L, $\beta 2-\mathrm{MG}$ 0.8-2.4, RDW-SD 35-56

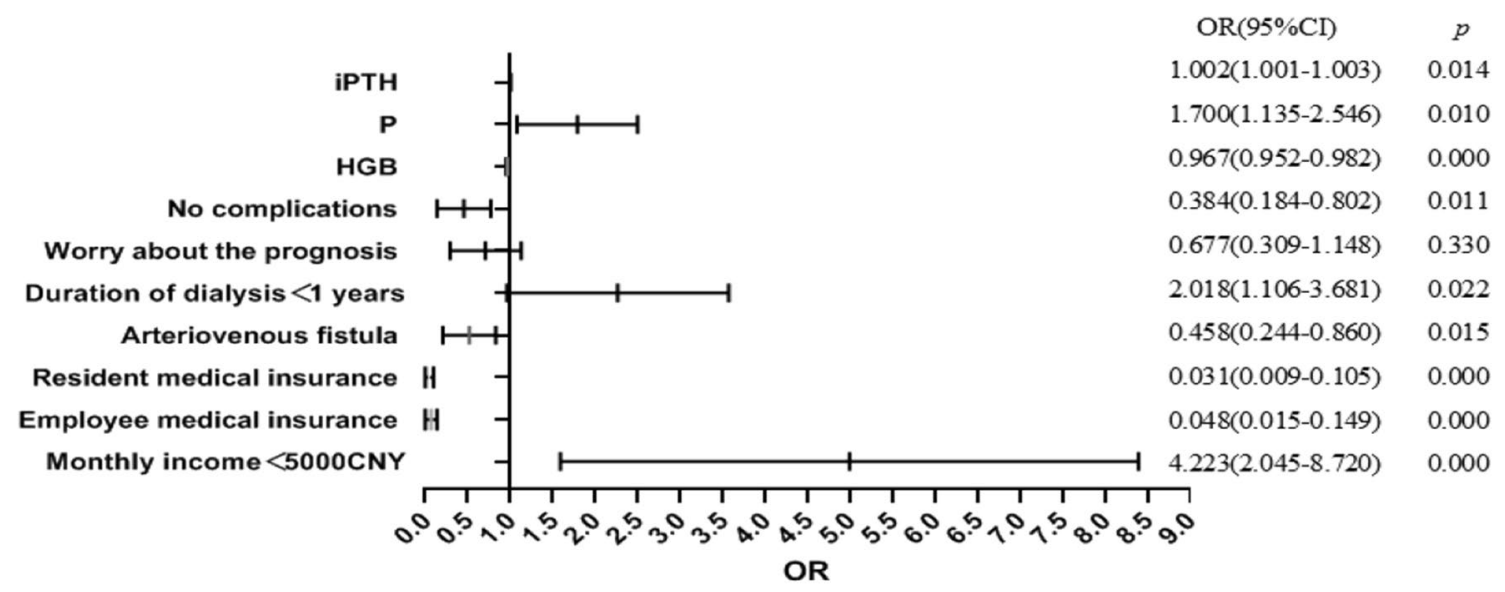

Fig. 5 Logistic analysis of anxiety symptoms among MHD patients $(N=321)$ 
stepwise regression analysis. The results showed that vascular access, medical insurance, monthly income, complications, Alb, P, and CRP were the factors that influenced depression (all $p<0.05$ ). Further, the risk of depression among MHD patients with a monthly income of less than 5,000 RMB was 3.562 times higher than that among patients with a monthly income of more than 5,000 RMB ( $\mathrm{OR}=3.562,95 \% \mathrm{CI}=1.625-7.809)$. Further, the risk of depression among patients with a resident medical insurance type was 0.072 times higher than that of patients with self-pay $(\mathrm{OR}=0.072,95 \% \mathrm{CI}=0.025-0.205)$. The risk of depression among the patients was 0.094 times higher than that of patients with self-pay $(\mathrm{OR}=0.094,95 \%$ $\mathrm{CI}=0.037-0.242$ ). The risk of anxiety among patients without health complications was 0.431 times higher than that among patients with health complications $(\mathrm{OR}=0.431,95 \%$ $\mathrm{CI}=0.202-0.921)$. Further, the risk of depression increased by $5.4 \%(\mathrm{OR}=1.054,95 \% \mathrm{CI}=1.005-1.106)$ for every unit increase in CRP in MHD patients, and by $63 \%(\mathrm{OR}=1.630$, $95 \% \mathrm{CI}=1.003-2.648$ ) for every unit increase in P. However, every unit increase in Alb reduced the risk of depression by $12.8 \%(\mathrm{OR}=0.872,95 \% \mathrm{CI}=0.805-0.945)$, as shown in Fig. 6.

\section{Discussion}

\section{Analysis of anxiety and depression symptoms of MHD patients during the COVID-19 outbreak}

Studies suggest that public health emergencies can substantially psychologically affect people. This can be expressed as anxiety, fear, and worry. In this study, we found a high prevalence of symptoms of anxiety and depression among YiBin MHD patients (between $34.89 \%$ and $32.09 \%$, respectively).
This is similar to the recent report made on health workers in China regarding cancer, diabetes, elderly patients, and the incidence of anxiety or depression among MHD patients during non-epidemic periods. However, it was evidently higher than that of the health workers in Nanjing (6.46\%) [5], Vietnamese outpatients (7.44\%) and the general population without quarantine in Chongqing (3.7\%) during the COVID-19 pandemic [6, 7]. This higher risk could be due to the coronavirus affecting the brain directly or indirectly by inducing a massive cytokine response [8]. In our sample, the prevalence of symptoms of anxiety and depression during the COVID-19 pandemic was between $34.89 \%$ and $32.09 \%$, respectively, within the range of the current global prevalence estimates for the general population (3.7-48.3\%) [9]. This was, however, higher than that of chronic kidney disease or end-stage renal disease patients in a meta-analysis (26.5\%) [10]. This may be because MHD patients are at higher risk of infection due to traveling to and from the dialysis center, and they feel that their immunity is low and that the prognosis may be poor. Additionally, according to the prevention and control measures, the hospital recommends that patients cancel their escorts (except in special circumstances), wear masks throughout the process, disinfect their hands, and try to avoid eating in the dialysis room. Implementing these restrictions may affect the psychological burden of patients.

\section{Analysis of the factors that influence anxiety and depression symptoms among MHD patients during the COVID-19 pandemic}

Yoong RK's [11] previous studies on end-stage renal disease patients show that dialysis patients are at risk of developing symptoms of anxiety and depression. The current studies indicate that this is the case with the COVID-19 pandemic.

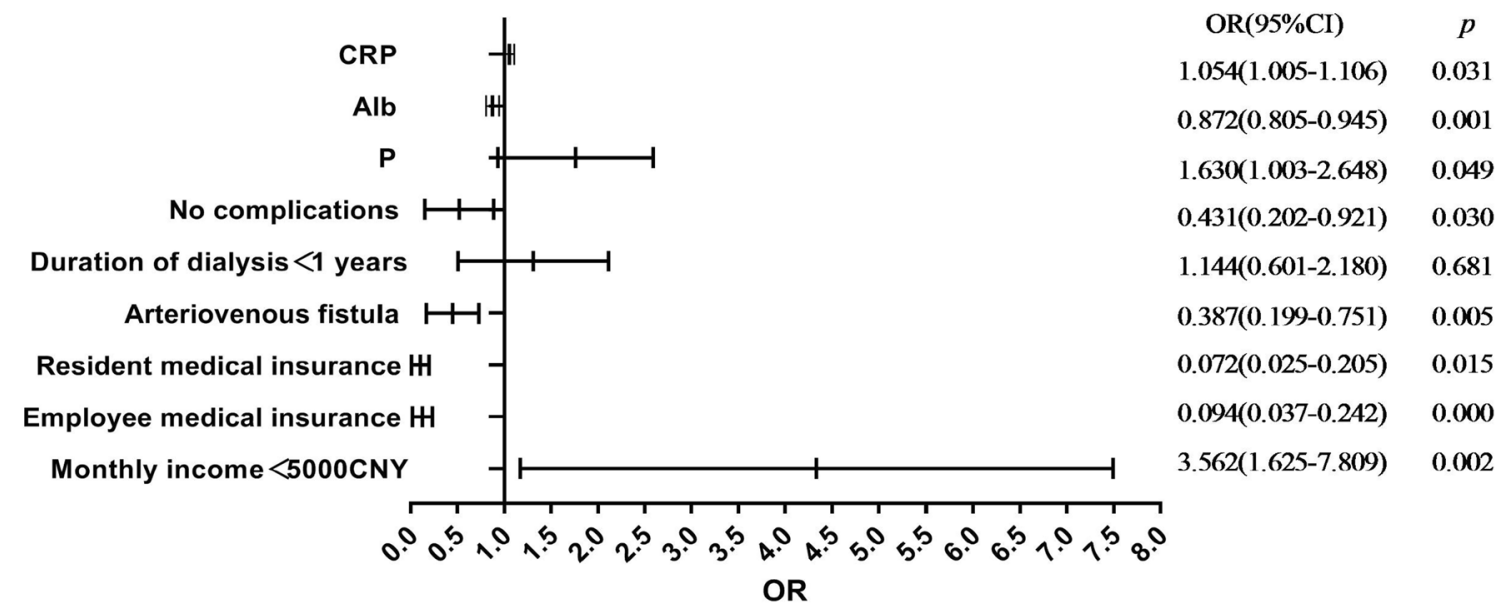

Fig. 6 Logistic analysis of depression symptoms among MHD patients $(N=321)$ 
Dialysis patients should be regarded as a highly susceptible group with a higher risk of psychiatric symptoms during the COVID-19 pandemic. Multivariate logistic regression analysis suggested that patients without complications, in contrast to patients with complications, was conducive to reducing the anxiety and depression of MHD patients. This might be explained as the complications may cause physical discomfort, and the need to take more medications and restrict certain activities, as shown in the study of Hou Y et al. [12]. Further, they also stated that comorbidities might increase the risk of the development of depression and anxiety. For instance, patients with symptoms of depression also had other comorbidities such as diabetes mellitus, hypertension, cardiovascular heart disease, hypoalbuminemia, and substance abuse. The results suggested that arteriovenous fistula, in contrast with catheterization, was effective in reducing the anxiety and depression of MHD patients. This might be explained by the concern of patients with catheters who state that their catheters are infected and that bathing is limited in their daily life.

Moreover, compared with self-pay mode, a resident medical insurance and employee medical insurance can help reduce anxiety and depression among MHD patients. This may be because self-paid patients are more worried about the economic burden of treatment costs on their families. Hou et al. [13] studied the factors associated with depression (SDS) and anxiety (SAS) among 81 patients with end-stage renal disease who were undergoing maintenance hemodialysis: the results showed that female patients were more likely to develop anxiety, whereas male patients were more likely to exhibit symptoms of depression. However, our research shows that during the epidemic, the difference in anxiety or depression between men and women is not statistically significant, which could be explained by home isolation during the pandemic.

The COVID-19-related stressors, which include iPTH, P, NLR, CRP, and ADL were positively associated with anxiety and depression symptoms among MHD patients during the pandemic, which is consistent with our hypothesis. Additionally, HGB and Alb were negatively associated with symptoms of anxiety and depression among patients. Anemia is a common complication among MHD patients. This assertion is consistent with the results of LIU X et al.'s [14] study. The 2012 KDIGO guidelines recommend that the target HGB be between 110 and $120 \mathrm{~g} / \mathrm{L}$. In this study, HGB exceeding $110 \mathrm{~g} / \mathrm{L}$ accounted for $37.38 \%$ samples. Further, more than $50 \%$ of the patients did not meet the target. The literature states that adverse cardiovascular events and all-cause mortality among patients decrease by between $6 \%$ and $5 \%$, respectively [15], for every $10 \mathrm{~g} / \mathrm{L}$ increase in HGB levels among MHD patients. Additionally, this study's results also indicate that a low HGB is a risk factor for anxiety. This might be because low HGB levels may cause patients to experience dizziness, palpitations, and fatigue. High HGB levels can improve the patient's exercise ability and meet the needs of the patients' daily activities. Therefore, it is important for MHD patients to keep their HGB levels within the target range. High levels of $\mathrm{P}$ and iPTH are also risk factors for patients with symptoms of anxiety. This may be because high phosphorus can easily cause symptoms of itching and secondary hyperparathyroidism, which can cause chronic kidney disease-mineral and bone disorder. Protein-energy malnutrition is another common complication among MHD patients, which is related to Alb. Evidently, the incidence rate of protein-energy malnutrition among MHD patients is $18-75 \%$. Further, Alb is one of the most commonly used indicators of nutritional status. According to Kalantar-Zadeh $\mathrm{K}$ et al. [16], when Alb is lower than $38 \mathrm{~g} / \mathrm{L}$, the mortality rate increases significantly. In our study, only $28.35 \%$ of patients had Alb within the normal range $(40 \mathrm{~g} / \mathrm{L})$. Additionally, our study also found that low levels of Alb were associated with symptoms of depression and showed the same trend as HGB. Thus, low Alb may be an independent risk factor for symptoms of depression, as indicated by LiFan [17], who found that the Alb of depressed patients was significantly lower than that of non-depressed individuals. Russell KL Yoong et al. [18] found that Alb was the only clinical parameter related to symptoms of depression. This may be due to the fact that that malnutrition weakened the patient's immunity and decreased activity. During the pandemic, news and the pressure of prevention, control policies, and the restriction of food purchases have caused certain psychological pressure on patients. This affects the quality of life of patients. Therefore, it is necessary to combine various methods and policies to improve the Alb level of patients.

\section{Limitations of the study}

(a) The study is cross-sectional and cannot provide a direct cause-and-effect risk association. (b) Our study is crosssectional hence, there are no longitudinal data regarding the burden of depression/anxiety during the spread of the pandemic. The burden of these complications may follow temporal variations. (c) During the investigation and research periods, China's policy was to block Wuhan and implement strict prevention and control measures across the country, such as wearing masks, quarantining at home, and controlling hospital attendants. The hospitals with dialysis patients were restricted by the local government, which might have affected the anxiety or depression status of patients.

\section{Summary}

COVID-19 is a serious threat to human health. A series of psychological problems have been triggered by the combined action of various factors during the pandemic. The 
current focus on the transmission of COVID-19 worldwide may probably distract the public's attention from the psychosocial consequences of the outbreak among affected individuals and the general population. The emerging mental health issues related to this global event may develop into long-lasting health problems. It is unfortunate that compared to other chronic illnesses such as coronary heart diseases and cancers, studies on MHD and related psychological disorders are far less extensive. Conclusively, global health measures should be employed to address psychosocial tension, particularly among MHD patients and other chronic diseases.

Acknowledgement This research received no specific grant from any funding agency in the public, commercial, or not-for-profit sectors.

Data availability All data and clinical data mentioned in this article are known to patients and their families, and they have signed an informed consent and agreed to use this information for the study.

\section{Compliance with ethical standards}

Conflict of interest The authors declare no conflict of interest in preparing this article.

Ethical approval This study was approved by the ethics committee of The Second People's Hospital of Yibin, and all the subjects signed the informed consent.

Consent for publication Informed written consent was obtained from the patient for publication of this manuscript.

\section{References:}

1. Cvc C, S-CTKKHPK-Y WWLY (2020) Preparedness and proactive infection control measures against the emerging novel coronavirus in China. J Hosp Infect 104:254-255

2. Huang C, Wang Y, Li X, Ren L, Zhao J, Hu Y et al (2020) Clinical features of patients infected with 2019 novel coronavirus in Wuhan, China. Lancet 395:497-506

3. Watnick SME (2020) On the frontline of the COVID-19 outbreak: keeping patients on long-term dialysis safe. CJASN 15:710-713

4. Dunstan DA, Scott N, Todd AK (2017) Screening for anxiety and depression: reassessing the utility of the Zung scales. BMC Psychiatry 17:329

5. Yang S, Ying C, Ning L, Chen T, Ming C, Ye-Wei Z et al (2020) Emergency responses to Covid-19 outbreak: experiences and lessons from a general hospital in Nanjing, China. Cardiovasc Inter Rad 43:810-819

6. Nh C, Nm H, Db N, Tc Q, Ntt P, Pk M et al (2020) People with suspected COVID-19 symptoms were more likely depressed and had lower health-related quality of life: the potential benefit of health literacy. J Clin Med 9:910-965

7. Wanqiu T, Fengyi H, MR S, LiXiaojiangLing JJZ et al (2020) Is returning to work during the COVID-19 pandemic stressful? A study on immediate mental health status and psychoneuroimmunity prevention measures of Chinese workforce. Brain Behav Immun 87:84-92

8. Te A, Kj N, Suzi H (2020) Are we facing a crashing wave of neuropsychiatric sequelae of COVID-19? Neuropsychiatric symptoms and potential immunologic mechanisms. Brain Behav Immun 87:34-39

9. Ahmed MZ, Ahmed O, Aibao Z, Hanbin S, Siyu L, Ahmad A (2020) Epidemic of COVID-19 in China and associated psychological problems. Asian J Psychiatry 51:10-1016

10. Silva ACSE, Miranda AS, Rocha NP, Teixeira AL (2019) Neuropsychiatric disorders in chronic kidney disease. Front Pharmacol 10(932): 10-3389

11. Yoong RK, Mooppil N, Khoo EY, Newman SP, Lee VY, Kang AW et al (2017) Prevalence and determinants of anxiety and depression in end stage renal disease (ESRD). A comparison between ESRD patients with and without coexisting diabetes mellitus. J Psychosom Res 94:68-72

12. Biswas S SMAS, (2014) Emergent median sternotomy for mediastinal hematoma: a rare complication following internal jugular vein catheterization for chemoport insertion-a case report and review of relevant literature. Case Rep Anesthesiol: 190110-190172

13. Hou Y, Li X, Yang L, Liu C, Wu H, Xu Y et al (2014) Factors associated with depression and anxiety in patients with end-stage renal disease receiving maintenance hemodialysis. Int Urol Nephrol 46:1645-1649

14. Tang X (2018) Analysis of anxiety, depression and influencing factors in maintenance hemodialysis patients. Anhui Medical University, Anhui

15. Alp IT, CNHaroldDenisJonathanKamyar JFFHK et al (2013) Prevention and treatment of protein energy wasting in chronic kidney disease patients: a consensus statement by the International Society of Renal Nutrition and Metabolism. Kidney Int 84:1096-1107

16. Ar J, Fk E, Cr E, Lp J (2001) The prevalence of comorbid depression in adults with diabetes: a meta-analysis. Diabetes Care 24:1069-1078

17. RAO W (2014) Clinical analysis of anxiety, depression and nutritional status in maintenance hemodialysis patients. Chin J Integr Med 15:886-888

Publisher's Note Springer Nature remains neutral with regard to jurisdictional claims in published maps and institutional affiliations. 